\title{
ESTUDIO DE CASO SOBRE LA APLICACIÓN DE UN PROGRAMA DE APRENDIZAJE CON TECNOLOGÍA 3D EN LAS AULAS DE EDUCACIÓN INFANTIL
}

\author{
Esteban Cabrera Noguera \\ Universidad de Cádiz \\ estebancabreranoguera@gmail.com \\ Noemí Serrano Díaz \\ Universidad de Cádiz \\ Juan Antonio Antequera Barroso \\ Universidad de Cádiz \\ Yésica Del Águila Ríos \\ Universidad de Cádiz \\ Jorge Guerra Antequera \\ Universidad de Extremadura
}

Fecha de Recepción: 22 Agosto 2019

Fecha de Admisión: 25 Septiembre 2019

\section{RESUMEN}

El mundo escolar ha tenido que hacer frente a numerosos desafíos como, por ejemplo, nuevos modelos de aprendizajes, nuevos procedimientos y estrategias didácticas.

Se presenta un estudio sobre la implementación de un programa de aprendizaje con el uso de las tecnologías emergentes 3D en las aulas de Educación Infantil.

La metodología utilizada tiene carácter mixto, es decir, con una metodología cuantitativa se procede a la evaluación de los resultados del programa de aprendizaje implementado, además de conocer, a través de un análisis cualitativo, la percepción del profesorado en la implementación del programa de enseñanza-aprendizaje y su formación acerca de las nuevas tecnologías.

El programa se ha llevado a cabo en un aula de tercer curso de Educación Infantil ( $\left.n^{\circ}=20\right)$ con alumnado de entre 5 y 6 años de edad.

Se obtuvo las siguientes conclusiones: la mayoría de los ítems analizados están por encima de la media, encontrando la diferenciación entre 2D al 3D como el ítem más bajo, y destacar como muy viable el uso de estas en edades tempranas, donde la mayoría de ellos se desenvolvieron sin mayores problemas. En cuanto a la información recabada sobre la percepción del profesorado, observamos como la mayoría de ellos estarían interesados en saber utilizar esta tecnología emergente, y destacaban que la necesidad de más formación en este ámbito.

Palabras claves: TIC; tecnologías 3D; educación; programa de aprendizaje. 


\section{ABSTRACT}

Case study on the application of a learning program with 3D technology in the children's education classrooms. The school world has had to face many challenges, such as new learning models, new procedures and teaching strategies.

A study is presented on the implementation of a learning program with the use of emerging $3 D$ technologies in the classrooms of Early Childhood Education.

The methodology used is mixed, that is to say, with a quantitative methodology the evaluation of the results of the implemented learning program is carried out, as well as knowing, through a qualitative analysis, the perception of the teaching staff in the implementation of the teaching program -learning and its training about new technologies.

The program has been carried out in a classroom of the third year of Early Childhood Education $\left(n^{0}=20\right)$ with students between 5 and 6 years of age.

The following conclusions were obtained: most of the items analyzed are above the average, finding the differentiation between $2 \mathrm{D}$ and $3 \mathrm{D}$ as the lowest item, and highlighting how very viable the use of these at early ages, where most of them They developed without major problems. Regarding the information collected on the perception of the teaching staff, we observed how most of them would be interested in knowing how to use this emerging technology, and highlighted the need for more training in this field.

Keywords: TIC; 3D technologies; education; training.

\section{INTRODUCCION}

La sociedad actual es una sociedad sorprendentemente cambiante en todos los aspectos, hemos pasado de la era industrial a la era de la información y la comunicación con una rapidez asombrosa. Vivimos en una sociedad denominada de la información. En los últimos años la sociedad está viviendo un desarrollo notable en las tecnologías de la información y la comunicación (en adelante TIC) en el campo de la educación. En un futuro, el proceso de enseñanza y aprendizaje se verá condicionado por nuevos usos. Sánchez (2016), señala que el siglo XXI se desenvuelve en un contexto marcado por nuevos retos y nuevas oportunidades en cuanto al desarrollo de la información, el conocimiento y el aprendizaje. Esto obligará tanto a los educadores como a los alumnos a familiarizarse con estas desde los primeros años de escolarización.

La forma de aprender de nuestro alumnado ha cambiado, sus necesidades y circunstancia no son las mimas que hace años atrás, por esta razón tanto por parte del centro educativo como por parte del profesorado, es del todo necesario, una formación continua en estas nuevas tecnologías y su uso en el aula. En esta misma línea Cejas, Navio y Barrso (2016) sostiene que "el docente es el responsable de diseñar tanto las oportunidades de aprendizaje como el entorno propicio que facilite el uso de las TIC por parte del alumnado" (p.106). Ante esto, la importancia de esta investigación es, en primer lugar, debido a la escasa participación de las TIC en el aula de infantil, tanto en la normativa como en el uso y fomento de estas en las aulas. En segundo lugar, por los beneficios que aportan, por un lado, aumenta la creatividad, la visión espacial, el reconocimiento de figuras geométricas, el trabajo en equipo y, por último, al iniciar en edades tempranas este acercamiento a las tecnologías emergentes, lograremos una mayor facilidad en la adquisición de conocimientos, habilidades, destrezas en el marco de las competencias digitales.

El mundo escolar ha tenido que hacer frente a numerosos desafíos como, por ejemplo, nuevos modelos de aprendizajes, nuevos procedimientos y estrategias didácticas, nuevas metodologías y nuevos recursos que faciliten la integración de las TIC en el proceso de enseñanza-aprendizaje (Roblizo y Cózar, 2015). La educación tiene un importante papel a desempeñar en relación con los avances tecnológicos y su uso como herramientas educativas. 
Este trabajo se centra en realizar un acercamiento a la tecnología emergente 3D para llevar a cabo la implementación de un programa de aprendizaje a través de esta tecnología y conocer lo que pueda aportar pedagógicamente.

Por otro lado, parece relevante conocer la percepción del profesorado acerca del uso del 3D en el aula, así como sobre la formación recibida para implementar las tecnologías en el aula. Conocer la opinión del profesorado, es fundamental por su papel crucial dentro del aula en el acompañamiento de los aprendizajes. Por todas estas razones, queda justificado el estudio que se presenta.

En esta investigación se pretende llevar a cabo en primer lugar una revisión teórica sobre el estado de las TIC en la etapa de Educación Infantil y conocer cómo se está trabajando actualmente. Para ello Martin-Laborda (2005) expone la importancia de "potenciar estas habilidades en edades temprana para que aprovechen al máximo las posibilidades que aportan las TIC al aula" (p.6). Es por esta razón por la que se debe empezar a potenciar estos usos desde el inicio de la escolarización en la etapa de Infantil. Además, se ha llevado a cabo una revisión teórica acerca de las políticas educativas a nivel nacional e internacional acerca de la implementación de las TIC y la formación del profesorado en competencias digitales en la etapa de Infantil. A través de los distintos autores, trataremos temas como la alfabetización digital y formación docente, siendo crucial la formación continua por parte del profesorado para poder llevarla de una manera eficaz al aula. De esta manera, el alumnado podrá estar actualizado en las nuevas tecnologías que le rodean desde de los primeros años.

Por último, llevar al aula las tecnologías 3D en esta etapa y conocer cuáles son los beneficios que pueden aportar a los alumnos, como la creatividad, el trabajo en equipo, la motivación, etc. Además, la impresión 3D es una tecnología que crece a gran velocidad en la industria y la ingeniería, la sanidad, la arquitectura, el mundo del diseño y, también, la educación.

Por todas estas razones en el presente artículo, se tratará de abordar los aspectos que rodean tanto al profesorado como al alumnado, con la implementación del programa. Este acercamiento se lleva a cabo, desde distintas perspectivas, una de ellas es la implementación de un programa de aprendizaje a través de la iniciación en las tecnologías 3D, la percepción del profesorado en las TIC y las tecnologías 3D, concretamente, en un aula de infantil. Dicha intervención la llevaremos a cabo en el Colegio concertado Argantonio, situado en la ciudad de Cádiz. Este es un colegio que apuesta por las TIC dotando todas las aulas con wifi, ordenadores, pizarras digitales, iPads, etc. En él se realizaron varias sesiones con el alumnado; por medio de actividades trataremos que obtengan una primera aproximación a estas tecnologías y que empiecen a diferenciar entre 2D y 3D. Para no romper con la dinámica de la clase y los ritmos de aprendizaje, las actividades estaban relacionadas con los temas o tópicos que están trabajando en el aula. Se han llevado a cabo una serie de sesiones en el aula de 5 años del Colegio Argantonio, con el fin de proporcionar a los alumnos/as un primer contacto con esta novedosa tecnología. Cuando hablamos de objetivos, hacemos referencia a las metas que pretendemos conseguir con la elaboración de esta investigación. Toda investigación parte de unos objetivos que marcan sus líneas de actuación. Se trata de conocer e indagar en la formación del profesorado acerca de las TIC, y el interés y la motivación que esto tiene en introducir una nueva tecnología en el aula. Un segundo objetivo, es proporcionar al alumnado una primera toma de contacto con las tecnologías 3D en un aula de Infantil, con la implementación de un programa de aprendizaje, donde a través de actividades vayan iniciándose, reconociendo y familiarizándose con la tecnología a fin de evaluar las sesiones de aprendizaje.

\section{MÉTODO}

El análisis de los resultados se ha abordado desde una metodología mixta. Para la evaluación del programa de intervención con el alumnado, se ha utilizado la toma de datos recogida en el cua- 


\section{ESTUDIO DE CASO SOBRE LA APLICACIÓN DE UN PROGRAMA DE APRENDIZAJE CON TECNOLOGíA 3D EN LAS AULAS DE EDUCACIÓN INFANTIL}

derno de campo y una rúbrica de evaluación sistemática en una escala del 1 al 6 con un análisis estadístico con el programa SPSS 21.

Para el análisis e interpretación de los datos recogidos se ha usado el programa estadístico SPSS 21, gracias a este programa he podido sacar la moda, la desviación típica en el cuestionario del profesorado.

\section{Participantes}

En el programa de intervención en el aula con la tecnología 3D, han participado en el estudio, 20 niños y niñas, de los cuales el 55\% (11) eran niñas y el 45\% (9) eran niños, entre 5 y 6 años, del Colegio Concertado Argantonio.

Respecto a la información recabada entre el profesorado, los participantes han sido un total de 8 profesores de Educación Infantil de segundo ciclo. A saber: 2 profesores de primer curso, 2 de segundo, 2 de tercero de segundo ciclo de Educación Infantil 1 especialista en pedagogía terapéutica y 1 profesor en prácticas. Como información recabada en los datos demográfico podemos decir que el profesorado se encuentra en la franja de 40 a 50 años (50\%), aunque la edad media es 39 años. Son docentes con bastantes años de experiencia (la oscilación en la experiencia es de entre 11 a 20 años como docente) a excepción de la chica de práctica que tiene la edad de 21 y apenas llega al año de experiencia. Otro de los datos de la muestra es que el 100\% de los participantes son mujeres. Esto entra dentro de la tónica general que se da en Educación Infantil: la mayoría son profesoras en esta etapa.

\section{Procedimiento}

Se accede al centro educativo con los consentimientos tanto del colegio como de los padres 0 tutores legales del alumnado. Se implementó el programa de aprendizaje entre el alumnado de 5 años de edad y se procedió a su posterior evaluación. Al profesorado participante se le administró un cuestionario. Se informó sobre la confidencialidad de los datos recogidos.

El instrumento usado para recoger la opinión del profesorado se trata de un cuestionario de elaboración propia denominado "cuestionario sobre la percepción del profesorado sobre el uso de las TIC en el aula". Consta de 10 ítems en una escala con valores oscilan entre 1 (nada en absoluto) y 6 (excelente). El objetivo de este cuestionario es analizar y recoger la percepción del profesorado a acerca de lo que ellos perciben de lo que son las TIC y las tecnologías 3D en el aula de infantil. Para ello se han respondido a 4 ítems relacionados con el tema que nos ocupa, tecnologías y 3D, acompañados de un cuestionario sobre datos demográficos.

\section{Resultados}

la Evaluación del programa de aprendizaje se ha llevado a cabo a través de una observación participante en cada sesión, recogiendo datos relevantes para la futura evaluación mediante una observación sistemática donde se ha ido cumplimentando la escala de valoración que se ha elaborada para la evaluación de las diferentes sesiones, esta se ha llevado a cabo de manera individual y se ha querido indagar un poco más sobre los resultados realizando un análisis estadístico descriptivo básico (moda, media, desviación típica).

La escala de valoración está compuesta por nueve ítems, con una escala, con valores comprendidos entre 1 y 6 , (1. Nada en absoluto; 2. Algo; 3. Suficiente; 4. Bueno; 5 . Muy bueno 6. Excelente). Para la evaluación en este estudio de caso, hemos empleado un proceso continuo a través de la observación, siendo esta una de las principales técnicas de evaluación en esta etapa educativa. 
Tabla 1. Escala Likert ítems observación

\begin{tabular}{|c|c|c|c|}
\hline Ítems & $M$ & Mo & $d t$ \\
\hline 1. Ha mostrado interés por las actividades & 5.25 & 6 & .829 \\
\hline 2. Ha colaborado e interactuado entre ellos & 4.8 & 5 & 1.122 \\
\hline $\begin{array}{l}\text { 3. Ha participado el alumnado de forma activa durante el } \\
\text { desarrollo de las actividades }\end{array}$ & 5.3 & 5 & .09 \\
\hline 4. Ha diferenciado el 2D del 3D & 4.4 & 5 & 1.113 \\
\hline 5. Utiliza el ordenador con cierta autonomía & 5 & 5 & .975 \\
\hline 6. Ha mostrado atención durante las actividades. & 5.25 & 5 & .887 \\
\hline 7. Crean diferentes figuras geométricas. & 4.95 & 5 & 1.160 \\
\hline 8. Manipulan adecuadamente los materiales y texturas. & 4.9 & 5 & 1.044 \\
\hline 9. Han realizado un buen uso de las Tecnologías & 5.15 & 5 & .852 \\
\hline
\end{tabular}

Para la interpretación de los datos recogidos se ha usado el programa estadístico SPSS 21. Los resultados la tabla 2 muestran en general unas altas puntuaciones, al estar todos los ítems por encima de la media (3). De este modo, la evaluación de las diferentes sesiones ha sido satisfactoria. Se destaca como el alumnado ha mostrado mucho interés en las actividades $(M=5,56)$. Además, el trabajo en equipo ha sido conseguido con éxito $(M=5,3)$, así como su atención $(M=5,25)$. A pesar de mostrar un valor por encima de la media, el ítem $4(\mathrm{M}=4,4)$ que hace referencia a la diferenciación de figuras 2D y $3 \mathrm{D}$ ha sido el que ha obtenido una puntuación más baja. Estos últimos resultados nos hacen reflexionar y proponer una posible modificación, como propuesta de mejora, de profundizar y enfocar el objetivo de discernir entre 2D y 3D más profundamente y apoyado en un mayor número de actividades.

En relación a la opinión del profesorado en la siguiente tabla se puede observar a partir de 4 la tendencia a la valoración positiva del profesorado (buenos, muy buenos y excelente). Destacamos como la media de todos los ítems y los cuatro últimos están por encima de la calificación de "bueno" en el cuestionario, siendo esto lo más aceptado por el profesorado. 
ESTUDIO DE CASO SOBRE LA APLICACIÓN DE UN PROGRAMA DE APRENDIZAJE CON TECNOLOGíA 3D EN LAS AULAS DE EDUCACIÓN INFANTIL

Tabla 2. Opinión del profesorado sobre el uso de las TIC en el aula de Infantil

\begin{tabular}{cccc}
\hline Ítems & $M$ & Mo & $d t$ \\
& & & \\
\hline 1. ¿Cómo crees que ha sido tu formación en TIC? & 3.25 & 4 & .707
\end{tabular}

2. ¿Considera que posees el conocimiento metodológico para abordar las TIC en el aula?

$\begin{array}{lll}3.63 \quad 4 & .518\end{array}$

3. ¿Conoces las Competencias digitales que debe tener un educador?

4. ¿Consideras necesario la formación continua en competencia digital para el profesorado?

5. ¿Conoces las Tecnologías 3D?

$2.13 \quad 1 \quad 1.356$

6. ¿Crees que es pertinente incluir esta tecnología en los centros educativo?

$\begin{array}{lll}4.13 & 4 & 1.808\end{array}$

7. ¿Incluyes las Tic en el aula?

$\begin{array}{lll}4.75 & 5 & .707\end{array}$

8. En caso afirmativo ¿Cómo es la actitud de los alumnos frente a las TIC?

9. ¿Y su motivación?

10. ¿Crees que ya viene con alguna formación/experiencias desde casa?

$\begin{array}{lll}4.13 \quad 4 & 1.458\end{array}$

\section{CONCLUSIONES}

Es importante destacar que los datos recogidos no son datos extrapolables a otros centros dado que se trata de un estudio de caso de un colegio concreto.

Respecto al desarrollo de las actividades y la posterior observación, hemos observado en los resultados, cómo la mayoría de los ítems, están por encima de la media, considerando estos resultados como muy satisfactorios. Destacando la implicación e interés de los alumnos por las actividades. Desde la primera sesión donde realice una toma de contacto con ellos y ver el punto de partida, la mayoría conocían bien las TIC. Donde hemos encontrado puntuaciones más bajas es en el "ítem 4" que hace referencia a la diferenciación entre el 2D y 3D. Al alumnado de 5 años les costaba discernir entre los objetos/imágenes en 2 dimensiones y los que están en 3D.

También destacar favorablemente el "ítem 9" que hace referencia al buen uso, hemos observado como la mayoría de ellos actuaban de una manera natural, incluso el uso del ordenador apenas tenía que explicarles nada. Conocen y trabajan perfectamente con ordenadores. 
En cuanto a los datos recogidos en la encuesta de la percepción de profesorado (véase anexo I), observamos como en la pregunta referente a si le gustaría saber utilizar las tecnologías 3D, todos mostraron una contestación afirmativa. También cabe destacar la pregunta sobre si se sienten seguro al utilizar el TIC en el aula, la mayoría comentaban afirmativamente, pero algunos dejaban comentarios como: "si, pero sé que puedo actualizarme de manera externa" o "si, pero sé que podría mejorar en algunos casos". Ante esto algunos me comentaron que intentarían realizar algún curso para actualizarse. Por último, reflejar el ítem 1, que hace referencia a la formación en TIC que han recibido, donde la mayoría lo refleja como "suficiente" y el ítem 5, el cual hace referencia a si conocían la tecnología 3D.

En definitiva, la puesta en marcha de esta intervención ha resultado una experiencia muy gratificante, como futuro educador y con resultados muy positivos. Su desarrollo no se ha visto interrumpido por ningún aspecto, de esta forma hemos podido llevar todas las sesiones propuestas y actividades con éxito a la práctica. Esto ha supuesto introducir una nueva metodologías y forma de trabajar las TIC y las tecnologías 3D.

Hemos alcanzado el objetivo general que era la iniciación en el uso de las tecnologías 3D, a través de una metodología lúdica, donde veíamos alumnos motivados e interesados por las actividades, coincidiendo con las palabras de Amar (2008) en relación a la alfabetización digital, que comentaba la importancia de que el alumnado posea dicha alfabetización para desenvolverse en el futuro de la sociedad del conocimiento. Estas conclusiones se apoyan en los resultados obtenidos en la evaluación individual del alumnado participante.

Ante esta situación vemos una clara evidencia e importancia de que el profesorado debe tener una formación continua, desde las instituciones provinciales dotar contenidos transversales sobre las TIC y reelaboración de las competencias de estas adaptada a las nuevas exigencias de la actualidad, y también la reestructuración de la normativa vigente la cual queda recorrido por hacer y mejorar en esta etapa ya que en la última modificación educativa (LOMCE) apenas ha existido cambios y referencia a las TIC en la etapa de Educación Infantil. Por tanto, se debería cambiar la mentalidad y ofrecer al alumnado la posibilidad de trabajar desde edades tempranas con las tecnologías para que experimenten y se inicien en ellas, ya que hoy en día, las TIC son un elemento importante y tienen una gran repercusión en nuestras vidas. Solo debemos tener en cuenta la forma en la que la utilizamos, debemos enseñar a los niños y niñas a usarlas correctamente.

\section{REFERENCIAS BIBLIOGRÁFICAS}

Amar, V. M. (2008). Tecnologias de la informacion y la comunicacion, Sociedad y Educacion. Tebar: Madrid

Cejas, R., Navío, A. \& Barroso, J. (2016). Las Competencias Del Profesorado universitario desde El Modelo Tpack (Conocimiento Tecnológico Y Pedagógico Del Contenido). Pixel-Bit. Revista de Medios y Educación, 49, 105-119.

Ley Orgánica 2/2006, de 3 de mayo, de Educación (L.0.E.). (B.0.E. no 106, 4 de mayo de 2006).

Ley Orgánica 8/2013, de 9 de diciembre, para la mejora de la calidad educativa. (B.0.E. n- 295 de 10 de diciembre).

Martín-Laborda, R. (2005). Las nuevas tecnologías en la educación. Fundación AUNA: Madrid.

Orden de 5 de agosto de 2008, por la que se desarrolla el Currículo correspondiente a la Educación Infantil en Andalucía (BOJA., no 169 de 26 de agosto de 2008).

Roblizo, M. y Cózar, R. (2015). Usos y competencias en tic en los futuros maestros de educación infantil y primaria: hacia una alfabetización tecnológica real para docentes. Pixel-Bit, 47, 23-39. doi: 10.12795/pixelbit.2015.i47.02. 
ESTUDIO DE CASO SOBRE LA APLICACIÓN DE UN PROGRAMA DE APRENDIZAJE CON TECNOLOGÍA 3D EN LAS

AULAS DE EDUCACIÓN INFANTIL

Sánchez, I. R. A. (2016). La Sociedad de la Información, Sociedad del Conocimiento y Sociedad del Aprendizaje. Referentes en torno a su formación. Anales de Investigación, 12(2), 235-243.254295. 